\title{
SUR L'IDENTIFICATION DES UNITÉS PHONOLOGIQUES DU CASTILLAN
}

\author{
F. H. H. KORTLANDT
}

\section{LA NOTION DE PHONEME}

Le problème fondamental de la phonologie est la réduction d'un complexe phonique continu à une chaîne d'unités discrètes successives, fonctionnellement relevantes et non divisibles en de telles unités plus petites. Le résultat de chaque analyse phonologique doit être considéré comme la conséquence immédiate de la signification attribuée explicitement ou implicitement à la notion de phonème. On peut choisir une définition de phonème et l'accepter en tant que base axiomatique de l'analyse ou bien tenter de trouver une procédure pour l'inférer de principes linguistiques plus généraux. La première possibilité est plus simple et permet d'éviter des considérations extra-phonologiques. Ainsi, il faut choisir une définition qui détermine un critère suffisant et inéquivoque pour la délimitation des unités phonologiques.

Il y a deux points de vue plus ou moins opposés que l'on rencontre partout dans la littérature linguistique contemporaine: la conception de D. Jones, qui accentue l'aspect physique ("a phoneme is a family of sounds in a given language which are related in character and are used in such a way that no one member ever occurs in a word in the same phonetic context as any other member"), ${ }^{l}$ et celle de N. S. Troubetzkoy, qui attache plus d'importance aux relations dans le système de la langue ("le phonème est la somme des particularités phonologiquement pertinentes que comporte une image phonique"). ${ }^{2}$

La définition de Jones détermine un critère double. En premier lieu elle contient une condition relative à la nature physique du son. Mais le fonctionnement des sons dans la langue ne dépend pas de leurs qualités physiques: au contraire, la réalisation physique d'un phonème est la

1 D. Jones, The Phoneme, Its Nature and Use (Cambridge, 1950), 10.

2 N. S. Troubetzkoy, Principes de phonologie, traduits par J. Cantineau (Paris, 1957), 40. 
conséquence de ses propriétés fonctionnelles et du contexte phonologique (si l'on fait abstraction de l'influence d'éléments stylistiques et psychologiques qui se superposent à la chaîne des unités relevantes du point de vue phonologique). En outre, l'attribut "related in character" ne spécifie pas un critère inéquivoque pour l'identification des phonèmes. Les sons $[p],[t],[k]$, ou $[\theta],[r],[\gamma]$, ou $[j],[1],[w]$ seront considérés par les parleurs de langues dans lesquelles la différence entre eux n'a pas de valeur distinctive comme des sons voisins, mais par ceux qui se servent de la distinction pour discerner des signes linguistiques comme des sons tout à fait différents, surtout si les phonèmes correspondants ont des propriétés distributives dissemblables. Pour eux, /o/ est une 'voyelle' et $/ \mathbf{r} /$ une 'consonne'. Il est possible de préciser le critère de "relatedness" en recourant à la fonction distinctive, mais il paraît que cette solution n'est pas conforme à l'intention de Jones.

L'autre condition de la définition de Jones se rapporte aux propriétés distributives du son. Le critère me semble inadéquat. Le phonème hollandais $/ \mathrm{r} / \mathrm{a}$ une variante roulée et une grasseyée qui sont interchangeables dans toute position: elles ont la même distribution et le choix entre elles ne dépend pas du contexte phonologique. Ce sont tout de même des variantes d'un seul phonème /r/ puisque la distinction entre elles n'est pas utilisée pour discerner des signes linguistiques.

Ainsi on arrive à la caractéristique essentielle du phonème: sa fonction distinctive. Définissons le phonème comme le faisait Troubetzkoy et essayons d'inférer de cette définition un critère suffisant et inéquivoque pour la délimitation des unités phonologiques. Appelons 'trait pertinent' toute caractéristique phonique susceptible de différencier toute seule deux unités significatives $;^{3}$ donc, les traits pertinents PERMETTENT de discerner des signes qui s'opposent paradigmatiquement. Cette susceptibilité peut être soumise à une vérification expérimentale et doit être distinguée de l'emploi effectif de l'opposition dans la chaine parlée, le rendement fonctionnel, qui peut être négligeable. En hollandais, par exemple, la combinaison de voyelle lâche $+|z|$ ne se rencontre que dans les mots puzzel /p^zal/ et mazzel/mazal/, empruntés récemment à d'autres langues. Le fait même que le mot anglais puzzle n'ait pas été identifié avec la chaîne /pözel/ ou /pssel/ démontre que la combinaison de phonèmes réalisèe dans ce mot était une chaîne admissible dans la langue au moment de l'emprunt. Le nouveau mot introduisit une nouvelle combinaison de phonèmes dans le discours, mais présupposait la présence de cette combinaison 
dans la langue; il est le résultat d'une expérience naturelle qui nous a donné la preuve que l'opposition phonologique existait dans la langue si le rendement fonctionnel de l'opposition etait zéro. Il est possible que la simulation de telles expériences nous offre un critère suffisant et inéquivoque pour la délimitation des champs de dispersion des phonèmes. ${ }^{4}$

Le critère unique pour l'identification des phonèmes est dans cette conception leur fonctionnement POTENTIEL, dont la signification est illustrée par A. Martinet de la manière suivante: "Le langage a pour l'homme un but qui est d'agir sur ses semblables. C'est un outil, d'une grande complexité certes, mais un outil tout de même, et si nous en voulons saisir la nature proprement linguistique, il nous faut l'examiner, comme nous le ferions de tout autre outil, en considérant les éléments qui en assurent le fonctionnement. C'est du point de vue de la fonction, et de celui-là seulement, que nous pouvons nous prononcer sur l'identité ou la non-identité des éléments linguistiques. Soit un outil, au sens courant et vulgaire du terme, comme la clé. Sa fonction est de fermer et d'ouvrir une porte. [...] Le serrurier pourra, s'il le juge bon, donner à l'anneau une forme particulière ou employer un métal ou un alliage autre que celui de l'exemplaire qui a servi aux mesures. Cela n'empêchera pas l'usager d'accrocher les deux clés, l'ancienne et la nouvelle, au même clou et de considérer qu'en pratique les deux clés sont interchangeables et identiques." ${ }_{5}^{5}$ Donc, si l'on a un complexe phonique fixé (la clé), il est possible de concevoir une procédure expérimentale qui révèle son fonctionnement dans un contexte phonétique fixé (le trou de serrure), et le résultat de cette expérience est décisif pour l'identification phonologique du son.

\section{L'IDENTIFICATION DES PHONEMES CASTILLANS}

L'identification des consonnes $/ \mathrm{p} /, / \mathrm{b} /, / \mathrm{f} /, / \mathrm{t} /, / \mathrm{d} /, / \theta /, / \mathrm{s} /, / \mathrm{k} /, / \mathrm{g} /, / \mathrm{x} /, / \mathrm{m} /$, $/ \mathrm{n} /, / \mathrm{n} /, / \mathrm{l} /, / \mathrm{H} /, \mathrm{r} /$ et des voyelles $/ \mathrm{i} /, / \mathrm{e} /, / \mathrm{a} /, / \mathrm{o} /, / \mathrm{u} / \mathrm{n}$ 'offre pas de difficultés particulières, ${ }^{6}$ mais l'existence des phonèmes $/ \check{c} /, / \overline{\mathbf{r}} /, / \mathrm{j} /, / \mathrm{w} /$ est discutable.

2.1. Dans son article classique sur l'interprétation monophonématique ou biphonématique de complexes phoniques A. Martinet écrit au sujet de l'affriquée [tš] du castillan: “[š] n'existe que dans ce cas, tandis que [t] se

4 Cf. C. L. Ebeling, "Some Premisses of Phonemic Analysis", Word 23 (1967), 122-37.

5 A. Martinet, La linguistique synchronique (Paris, 1968), 60-61.

- Cf. Alarcos Llorach, 1965: 145-50 et 161-62. 
rencontre fréquemment dans bien d'autres positions; dans un mot comme chato [tšato], [š] est commutable puisqu'en le remplaçant par [r] on obtient le mot trato, et en le commutant avec zéro on obtient le mot tato. Mais, tandis qu'on peut commuter le [a] qui suit [š] et obtenir le mot choto, on ne peut ni supprimer le [t] qui le précède, ni le remplacer par rien sans obtenir des formes [šato], [kšato], [lšato], etc., qui sont impossibles en castillan. Le [t] du groupe [tšs] n'a, en lui-même, aucune valeur distinctive particulière, son apparition dans ce cas étant automatiquement déterminée par celle de [š]; [tš] est donc en castillan la réalisation d'un phonème $/ \check{c} /$. On pourrait être tenté de considérer le [̌̌] du groupe [ťs] comme une variante combinatoire de /s/ dont l'articulation castillane est assez voisine. Mais il faudrait pour cela que le voisinage de $[\mathrm{t}]$ justifie le caractère proprement chuintant de [š], caractère qui le distingue de [s], ce qui n'est pas le cas." On peut remarquer que: (1) l'argument est mal fondé, puisque le caractère phonétique des sons est irrelevant pour l'identification des phonèmes du point de vue de leur fonctionnement dans la langue; (2) l'argument est inadéquat: si Martinet s'était servi d'une transcription phonétique plus précise, il n'aurait pas observé que "[̌̌] $n$ 'existe que dans ce cas, tandis que [t] se rencontre fréquemment dans bien d'autres positions", puisque la variante prédorsoalvéolaire du son [t] qu'on trouve dans le mot [tšato] est nettement distincte de la variante apicodentale dans les mots [trato] et [tato]. Il semble que la distinction entre [s] alvéolaire et [s] palatal et la parité de [t] dental et [t] alvéolaire soient suggérées par le système phonologique d'autres langues européennes. En outre, le critère que "le voisinage de $[t]$ justifie le caractère proprement chuintant de [š]" est inapplicable, comme le montrent les exemples suivants.

(a) En japonais la réalisation phonétique de $/ \mathrm{n} /$ à la fin du mot est [n], par exemple rōjin [n], emprunté au chinois lăorén 'vieillard'. Le même phénomène peut être observé dans quelques parlers asturiens. Le caractère vélaire de la consonne nasale ne peut pas être expliqué par le caractère du phonème suivant. (Selon la théorie de R. Jakobson, ${ }^{8}{ }^{8}$ 'existence de [n] présuppose même l'existence de $[\mathrm{n}]$ et [m], ce qui est clairement erroné.)

(b) M. V. Panov donne les règles suivantes pour la prononciation littéraire du russe contemporain: ${ }^{9}$

7 A. Martinet, "Un ou deux phonèmes?", Acta Linguistica 1, 97; aussi dans 1968: 112-13.

8 R. Jakobson, Child Language, Aphasia, and Phonological Universals (The Hague, 1968), 53: "The existence of back consonants in the languages of the world presupposes accordingly the existence of front consonants."

9 M. V. Panov, Russkaja fonetika (Moskva, 1967), 92-96. 
- les consonnes apicales sont mouillées si elles précèdent une consonne labiale mouillée, par ex. zmeja [ẓmunáa] 'serpent';

- les consonnes labiales sont dures si elles précèdent une consonne apicale mouillée, par ex. ptica [pțítsa] 'oiseau'.

La réalisation mouillée (dure) de l'archiphonème peut être 'expliquée' par l'influence assimilatrice (dissimilatrice) du phonème suivant: l'origine de l'assimilation se trouve dans la tendance économique du système et l'origine de la dissimilation dans la nécessité de maintenir les contrastes qui permettent de distinguer les éléments successifs du discours. Mais ces explications se contredisent nettement et pour cela ne peuvent pas être suffisantes. Il s'ensuit que le caractère ambigu de l'influence de phonèmes voisins sur les caractéristiques phonétiques de la réalisation d'un phonème ne permet pas d'utiliser de manière inéquivoque l'argument du voisinage syntagmatique. En outre, il y a une objection plus fondamentale: le terme 'influence' n'a pas de valeur bien définie dans la linguistique synchronique. La notion d'influence présuppose la succession de cause et de conséquence, qui ne peut s'effectuer que dans un système dynamique, c'est-à-dire dans un système dont l'état est dépendant du temps, comme, par exemple, l'évolution d'une langue. Mais dans le système synchronique d'une langue les propriétés d'un élément ne peuvent pas être considérées comme la conséquence des propriétés d'autres éléments parce que toutes ces caractéristiques sont déterminées conjointement par l'évolution antérieure du système. On peut décrire le système ou considérer ce qui influence son évolution ou sa réalisation dans la parole, mais le système synchronique ne s'explique pas par soi-même.

On peut résoudre le problème de l'interprétation monophonématique ou biphonématique au moyen d'une procédure de vérification expérimentale. Quand il s'agit du groupe [tš] en castillan, l'exemple suivant, résultat d'une expérience naturelle, montre qu'il est phonologiquement distinct du groupe [ts], qui peut être considéré comme la réalisation de la combinaison de phonèmes /Ts/:

cheque [tšéke] 'chèque', tsetsé [tsetsé] 'tsé-tsé', queche [kétše] 'galiote'.

L'emprunt témoigne de l'existence de l'opposition phonologique au moment où le mot fut emprunté et la distinction dans la langue actuelle prouve que l'opposition se maintient. De la même maniere on prouve la distinction de [tš] et [ks] (sexo: hecho), etc. Ce n'est que pour cette raison qu'il y a un phonème /č/ en castillan. 
$\mathrm{Au}$ moyen-âge les conditions étaient différentes. Il existait un phonème $/ \check{s} /$, et pour cela le groupe [tš] doit être considéré dans le système médiéval comme la réalisation de /Tš/, à moins qu'on ne puisse prouver l'existence

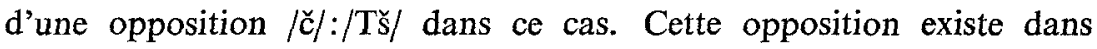
quelques langues, en russe par exemple:

$$
\begin{aligned}
& \text { lučšij [túț̣soì] /lúTšlj/ 'meilleur', } \\
& \text { letučij [lutúţ̦ṣ̌̂i] /ļItúčlj/ 'volant'. }
\end{aligned}
$$

Cependant, beaucoup de russes ne distinguent pas les membres de cette opposition et prononcent [łúţš̆in]: ils ont, par conséquent, un système phonologique sans / č/, que l'on ne doit pas confondre avec le système de la langue littéraire. De même, les espagnols qui prononcent [š] dans des noms étrangers disposent clairement d'un phonème / $/ \mathrm{s} /$ et n'ont donc pas besoin d'un phonème / $/$ / dans leur système phonologique. La situation est assez compliquée en polonais, où l'existence d'une consonne affriquée /č/ ne peut être établie que par une procédure expérimentale. Si les mots

$$
\begin{aligned}
& \text { patrzy [páț̦șt] /paTši/ '(il) regarde' et } \\
& \text { paczy [páț̦̌s] /pači/ '(il) déjette' }
\end{aligned}
$$

ne se confondent pas, l'affriquée existe, tandis qu'elle n'existe pas s'ils se confondent. La vérification expérimentale est nécessaire parce que la différence phonique peut étre suggérée par l'orthographe.

Ces observations éclaircissent que le système phonologique que l'on décrit est le système d'un individu (l'informant) et que des personnes qui 'parlent la même langue' peuvent malgré cela disposer de systèmes phonologiques différentes. La coexistence de tels systèmes se rencontre même toujours quand une langue évolue. (Il ne faut pas confondre la diversité de systèmes phonologiques avec la dissemblance de réalisations phonétiques du même système: le premier est un trait fonctionnel tandis que le second se rapporte à la parole.)

2.2. Quant à l'opposition /i/ : /j/ en castillan E. Alarcos Llorach écrit: "Sólo es válida la distinción en posición intervocálica: raía / raya, y entre consonante y vocal nuclear: desierto / deshielo. En todos los demás casos las variantes de /i/ y /y/ se complementan."10 L'argument est irrelevant à mon avis. Les mots raía et raya ne se distinguent que par le prosodème, qui est un trait configuratif en castillan. ${ }^{11}$ Le contraste syntagmatique ainsi établi permet d'opposer paradigmatiquement des signes à structures

10 Alarcos Llorach, 1965: 183.

11 Cf. C. L. Ebeling, "On Accent in Dutch and the Phoneme/o/", Lingua 21 (1968), 135. 
prosodématiques différentes. Dans cette conception la relation entre /raía/ et/ráia/ est la même qu'entre/sabána/ et/sábana/. L'opposition /i/ : /j/ n'existe pas en castillan parce que *ráia et raya ne se distinguent pas. (La syllabe n'est pas une entité phonologique en castillan.)

Les mots desierto et deshielo ou abierto et abyecto se distinguent au moyen d'une jointure (sutura) de morphèmes, signal démarcatif qui n'a pas de fonction distinctive (comme on peut le démontrer expérimentalement). Si l'on fait usage de tels phénomènes sans la vérification expérimentale de leur nature phonologique, on peut 'prouver' presque tout, cf. le fameux exemple russe:

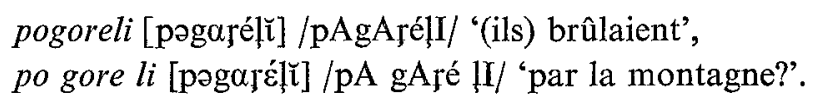

Il n'y a aucun doute que l'opposition $/ \mathrm{e} /: / \varepsilon /$ n'existe pas en russe.

Dans quelques cas Alarcos Llorach interprète le son [j] 'morphonématiquement' comme la réalisation de /ji/, par exemple dans les mots huyó et huyeron. ${ }^{12}$ L'argument n'est pas seulement mal fondé du point de vue théorique ${ }^{13}$ mais aussi inadéquat, comme la différence entre dirigieron /dirixiéroN/ et dijeron/dixéroN/ le montre. En effet, /éroN/ est la forme du morphème /iéroN/ après une consonne historiquement palatalisée: on dit salieron et vinieron mais bulleron et bruñeron.

Le son [w] doit également être considéré comme une variante du phonème $/ \mathrm{u} /$. Si de huellas et degüellas ne se distinguent pas, la transcription phonologique sera respectivement /de uélas/ et /deuélas/, quoique la réalisation phonétique puisse être [derwélas] dans les deux cas. L'élément $[\gamma] \mathrm{n}$ 'a apparemment pas de fonction distinctive dans cette combinaison. ${ }^{14}$ On pourrait aussi citer:

son nuevos [sonwéßos] /soN nuébos/, son huevos [sonwéßos] /soN uébos/, la suela [laswéla] /la suéla/, las huela [lazwéla] /las uéla/.

2.3. Pour établir l'existence du phonème $/ \overline{\mathrm{r}} /$ en castillan il faut démontrer qu'il ne peut pas être interprété comme une combinaison de phonèmes, en particulier qu'il se distingue de la combinaison /rr/. Alarcos Llorach ne voue pas plus d'une note à cette possibilité: “Algunos autores 


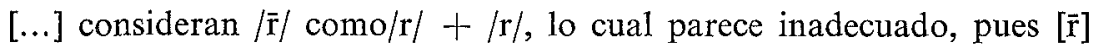
aparece en inicial, donde nunca hay consonantes dobles." ${ }^{15}$ L'argument ne tient pas puisque l'assertion qu'il n'y a pas de consonnes doubles en position initiale doit être prouvée par l'analyse phonologique et ne peut pas être considérée comme une condition axiomatique imposée a priori. Cela n'empêche pas que l'assertion est vraie, parce que [ī] en position initiale n'est pas la réalisation de $/ \mathrm{rr} /$ mais de $/ \mathrm{r} /$. Le caractère phonétique de $/ \mathbf{r}$ / initial est un signal démarcatif qui n'a pas de fonction distinctive, comme on peut le vérifier expérimentalement. La combinaison de phonèmes $/ \mathrm{rr} /$ ne se rencontre qu'en position intervocalique.

Je conclus ces remarques par un tableau du système consonantique du castillan actuel. ${ }^{16}$

$\begin{array}{llllll}p & b & f & m & & \\ t & d & \theta & n & l & r \\ \check{c} & & s & n & l & \\ k & g & x & & & \end{array}$

Université d'Amsterdam

15 Alarcos Llorach, 1965: 163.

${ }_{16}$ Cf. les tableaux dans B. Pottier, Phonétique et phonologie espagnoles (Paris, 1965), 86, et Alarcos Llorach, 1965: 170. 